\title{
Mabutsane Sub District Tuberculosis Program Performance from 2013 to 2015
}

\author{
Article by Kazadi Mantanki Emmanuel \\ General Practitioner, Congo \\ E-mail: emkazadi18@gmail.com
}

\begin{abstract}
A retrospective research through review of Tuberculosis (TB) registers was conducted in Mabutsane sub district from July to September 2016. This study was intended to assess the TB program achievements from the period of 2013 to 2015 in order to improve TB case management for better district Tuberculosis performance.

To this end, the cure and success rates, the proportion of TB patients with completed treatment, the defaults, as well as the HIV testing rates have been determined.

The results were as follows: the cure rates $29.57 \%$ (2013), 19\% (2014), 11.10\% (2015), the treatment completion $80 \%$ (2013), $79.4 \%(2014), 68.5 \%$ (2015), and the default rate was below $5 \%$ for the whole study period. The success rates were $78.6 \%$ (2013), 78\% (2014) and 67.1\% (2015).

The overall cure rate was low, and very low among patients seen in 2015. There have been a marked completion treatment and success rate over the first two years of a study with decline in 2015 due to poor documentation or poor program.

HIV testing rate was more than $90 \%$ among Tuberculosis patients throughout the all years, with TB/HIV co infection rate between $40 \%$ and $60 \%$.

Mabutsane sub district Tuberculosis program is a weak program as shown by a low cure rates, and sputum follow up.
\end{abstract}

Keywords: Tuberculosis cure and success rates, HIV testing rate, default rate, treatment completion.

\section{Introduction}

Tuberculosis is one of the major worldwide public health concerns, with approximately 9 million of new TB cases notified annually. Regionally, the southern Africa includes some world's high TB burden countries and has experienced the rise of TB cases for the recent years, mainly driven by highest HIV prevalence and poor socio-economic conditions. Thus, accounting for a large share of the global tuberculosis (TB) burden. In addition TB has become a cross-border issue in the southern Africa $(4,14)$.

Though it is curable and preventable, every day TB kills nearly 5,000 people (WHO, 1999, 2001, 2008), and represents the second leading cause of death from infectious diseases after HIV. Without treatment, the morbidity and mortality rates from TB are high, mostly in low resources setting countries.

Taking into account the high proportion of TB patients in HIV prevalence population of sub Saharan particularly the southern Africa, where studies have shown that for each increase of $1 \%$ of HIV prevalence, there is an increase in TB notification rates of 13 cases $/ 100,000$, and considering Botswana as one of the highest TB notification rates per capita in the world(6) and also our professional experience on TB case management; there was a pressing need to assess the TB program performance from 2013 to 2015 at the Mabutsane sub district level in order improve the district TB program performance.

To this end, the following questions have to be addressed:

- What is the TB cure rate?

- What is the percentage of people who completed TB treatment?

- What is the default rate?

- What is the TB success rate? 
DOI: $10.21522 / \mathrm{TIJPH} .2013 .05 .04 . A r t 036$

ISSN: $2520-3134$

- How many TB patients tested for HIV?

\section{Materials and methods}

This descriptive study was done retrospectively for the period of July to September 2016. It includes the data of interest from 2013 to 2015, and explored Mabutsane sub district TB program achievements. it was carried out on various key program indicators which include but not limited to the treatment outcomes of registered patents, treatment group ( new and subcategories of previously treated patients or retreatment TB), HIV status. TB patients transferred out of the district are also part of this study. Patients with multidrug resistance were excluded, as well as those who had unknown treatment outcomes.

- Reports from national TB program are also not part of the study.

- Data was collected electronically and manually from Mabutsane sub district TB register.

- Data was reviewed and presented as tables, graphs and charts.

Mabutsane is a rural sub district situated in the Southern district of Botswana along the Ghanzi road, approximately $300 \mathrm{~km}$ from Gaborone (Capital City of Botswana). Its health facilities include 5 clinics, 4 health posts, 2 mobile stop, one laboratory with sputum smear microscopy, Gene xpert, and integration of TB and community based care services. Directly Observed Therapy option for smear-positive Pulmonary TB patients for health facility supervision. Each facility provides TB treatment. There is no hospital in the district.

\section{Results}

Descriptive analyses were performed using percentages (proportions).

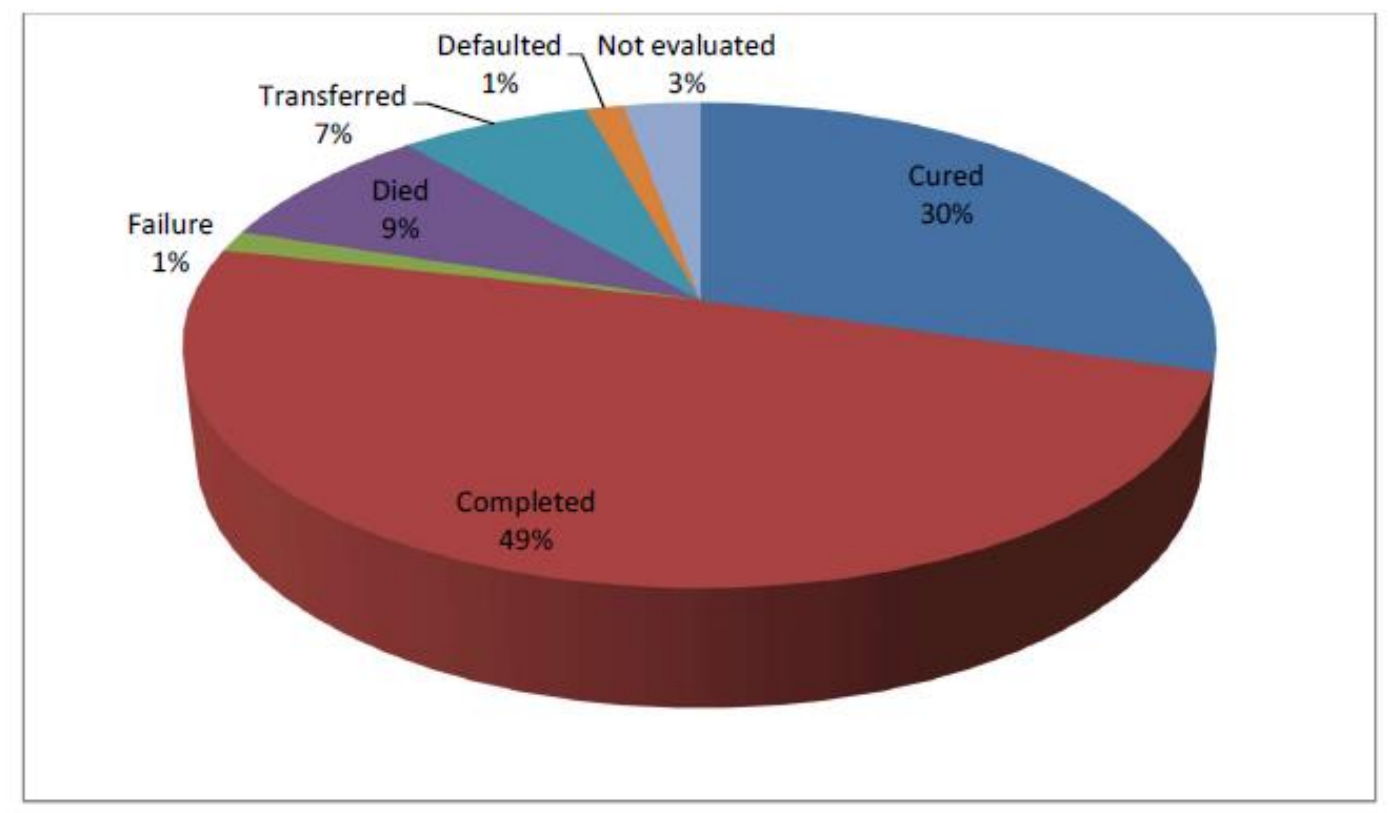

Diagram 1. Summarized treatment outcomes, 2013

This pie chart shows the proportion of $80 \%$ of TB patients who completed treatment. Of these, $30 \%$ were cured and $49 \%$ have completed treatment but not evaluated for sputum smear status at the end of treatment, and $1 \%$ represents the treatment failure.

At least $17 \%(12 / 70)$ of patients did not complete treatment. Of these, $1 \%$ of patients' defaulted treatment, $7 \%$ died, and $9 \%$ transferred out. However 3\% were not evaluated for treatment outcomes. 
The treatment success rate, which is the sum of the patients who were cured and those who completed treatment but not evaluated for sputum status throughout the course of treatment, is $78.6 \%(30+48.6)$. This is well explained in the following diagram:

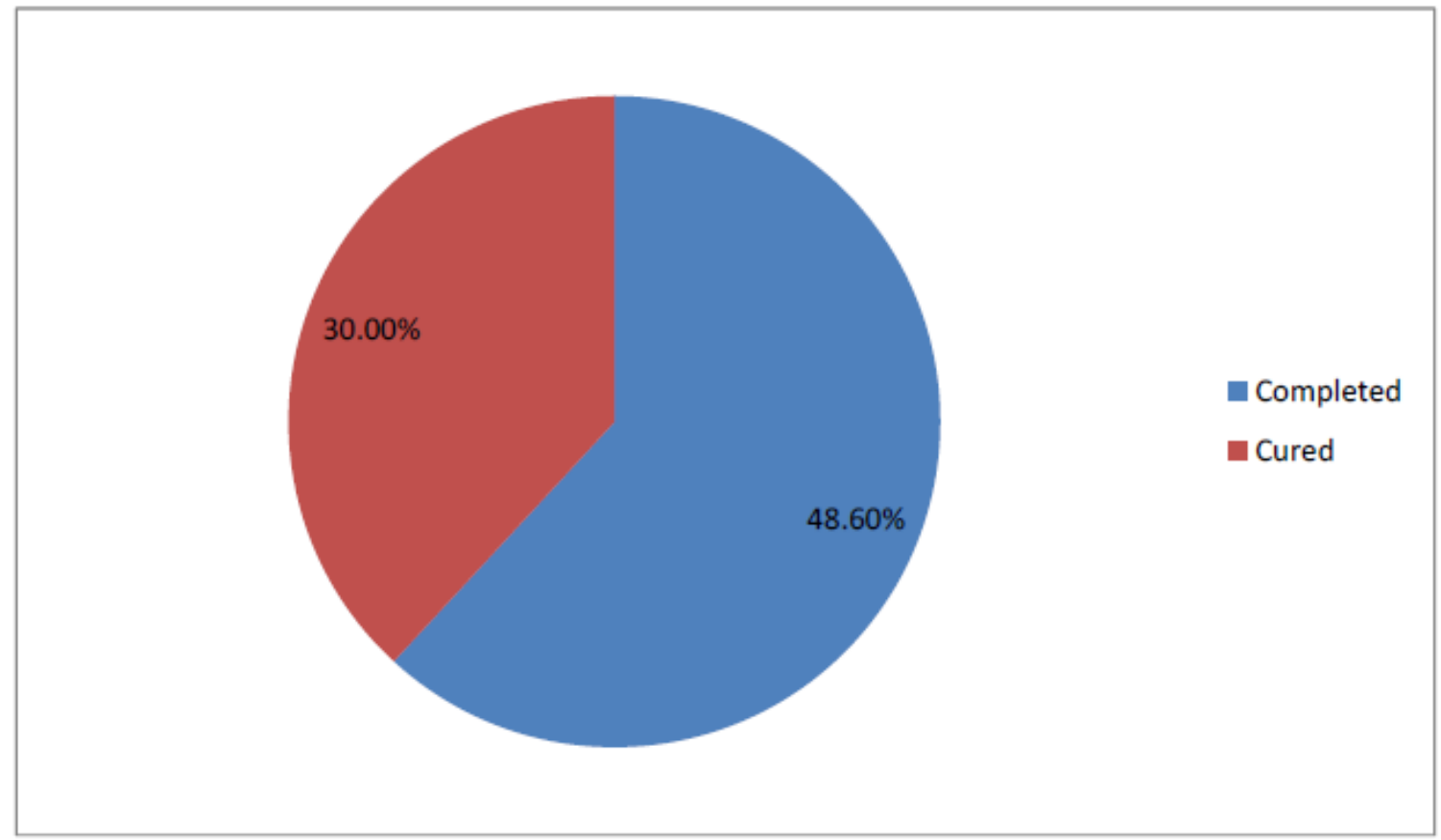

Diagram 2. Treatment success rate in 2013

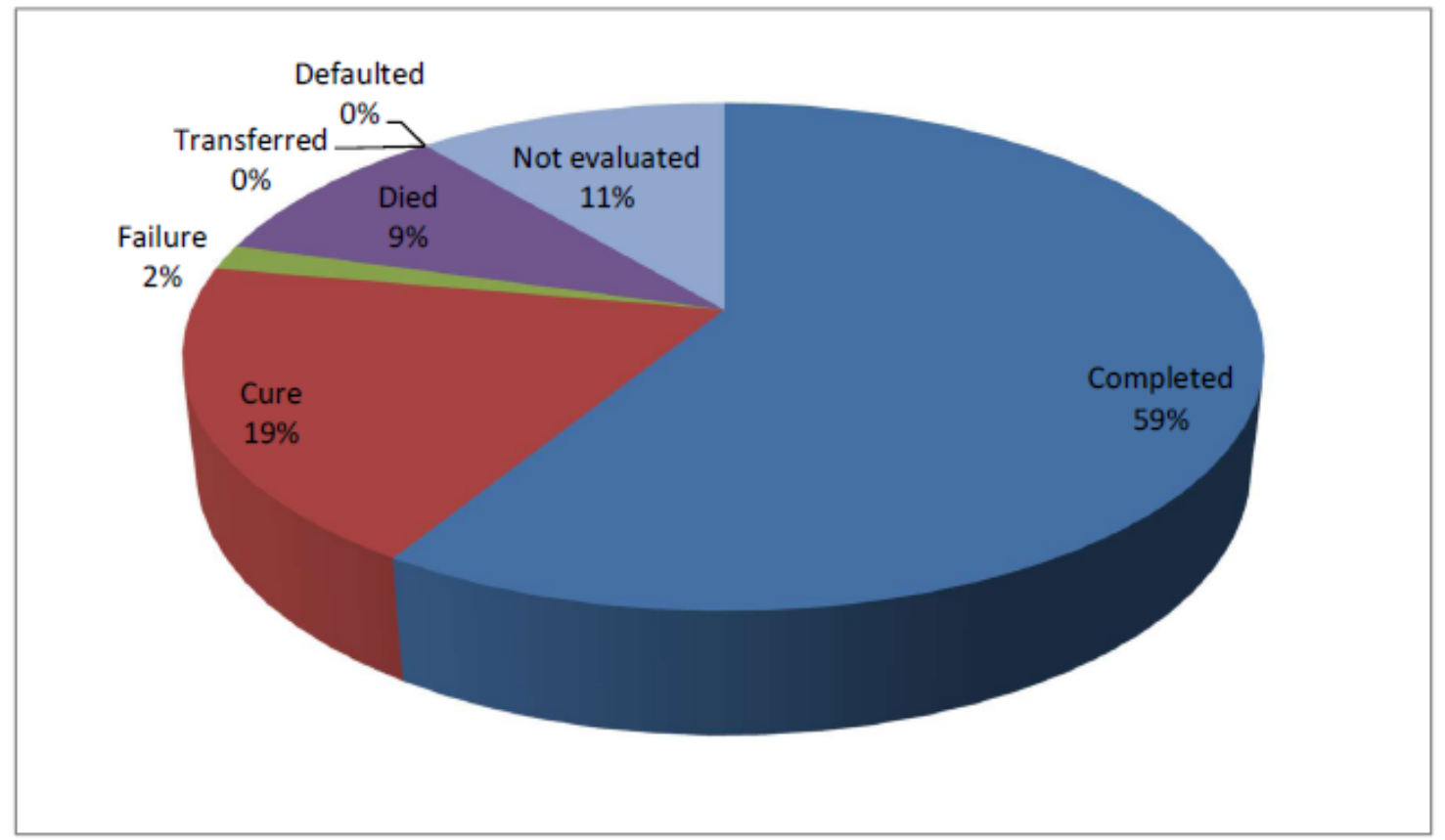

Diagram 3. Summarized treatment outcomes, 2014

This pie chart shows that $19 \%$ of patients were cured, $59 \%$ have completed but were not evaluated for sputum status at the end of treatment, and $2 \%$ of treatment failure. 
DOI: $10.21522 / \mathrm{TIJPH} .2013 .05 .04 . A r t 036$

ISSN: $2520-3134$

There were $9 \%$ of patients, the proportion of patients who did not complete treatment mostly those who died. The default rate was $0.0 \%$, and $11 \%$ of patients were not evaluated for treatment outcomes.

There was no patient transferred out in this year under evaluation.

The success rate for 2014, which is the sum of the cure rate of $19 \%(12 / 63)$ and the treatment completion of $59 \%(37 / 63)$ with no sputum evaluation at the end of treatment is $78 \%(19+59)$. This has been indicated summarized in the below diagram:

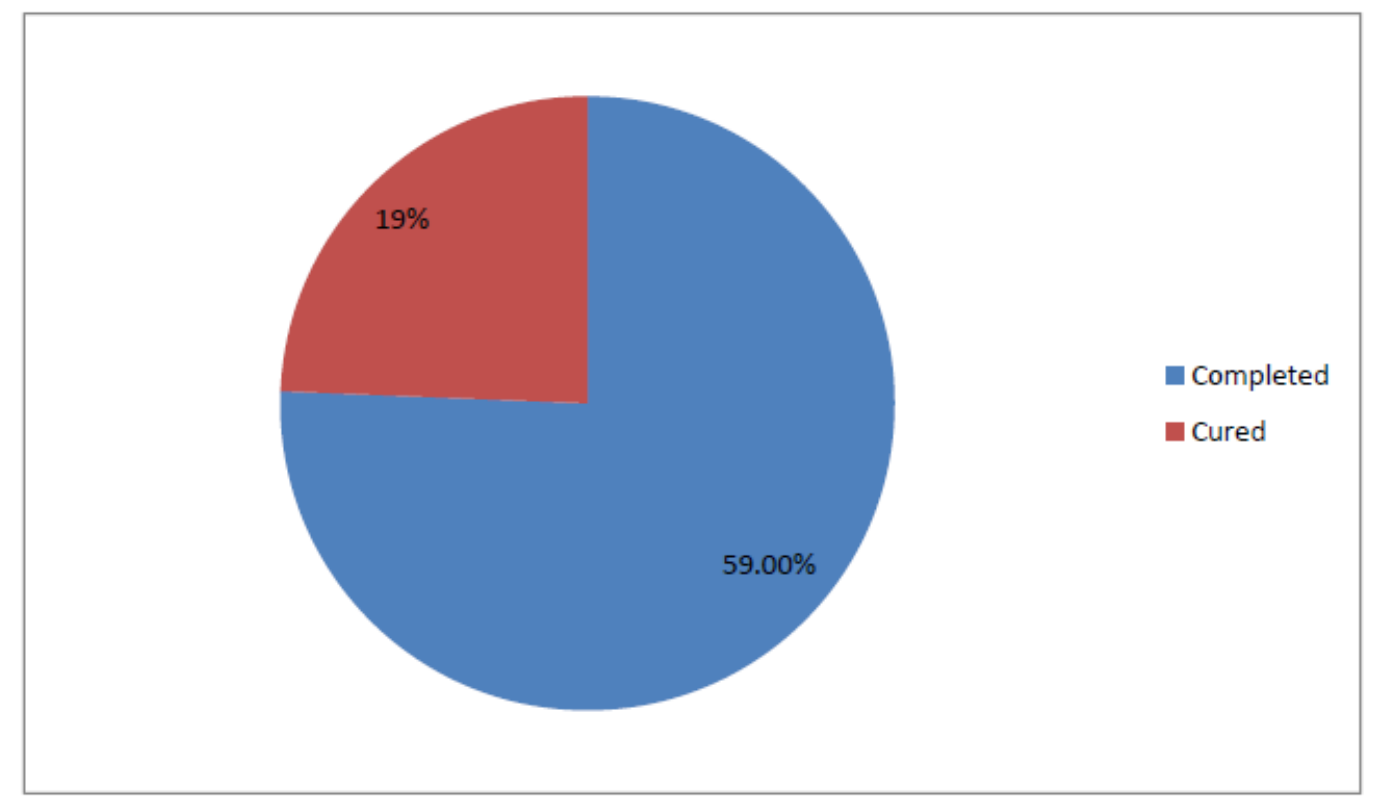

Diagram 4. Treatment success rate, 2014

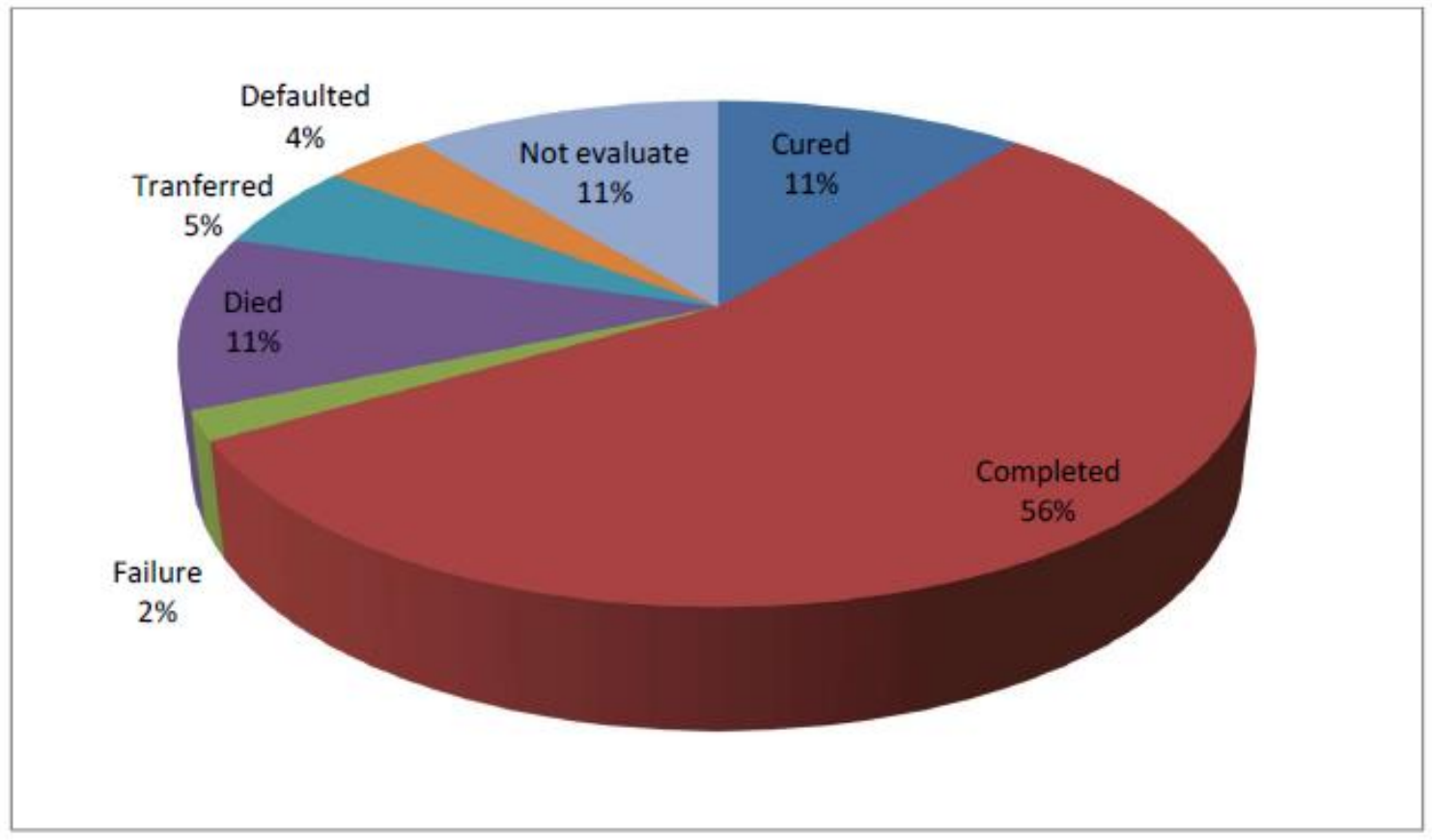

Diagram 5. Summarized treatment outcomes, 2015

Treatment completed with no sputum evaluation in 2015 was 56\%; cure rate $11 \%$, and treatment failure $2 \%$. 
Treatment not completed includes default (4\%), death (11\%), and transferred out (5\%).

$11 \%$ of patients were not evaluated

The success rate is the sum of treatment completion that results in $67.1 \%(56 \%+11.1 \%)$. It is summarized in the following diagram

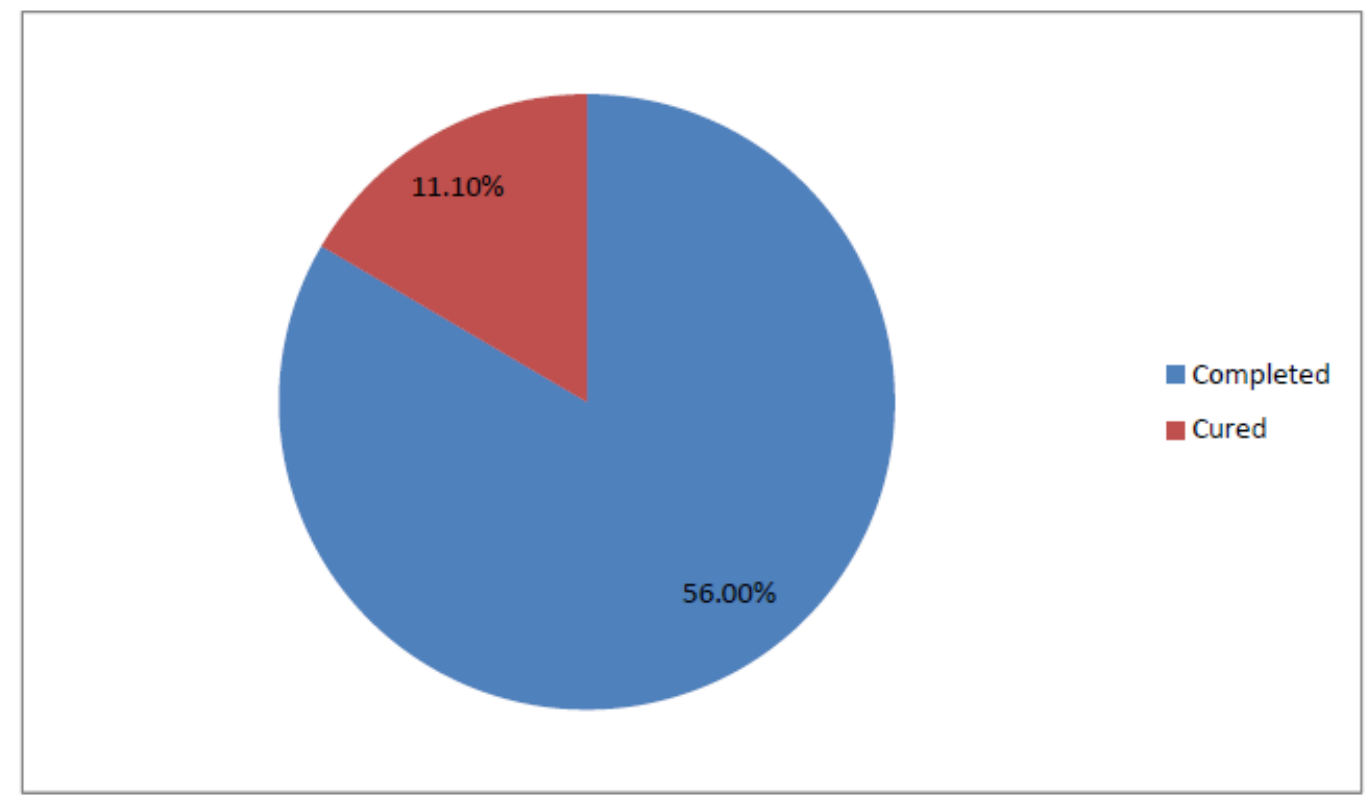

Diagram 6. Treatment success rate, 2015

This diagram indicates a success rate of $67.1 \%(11.1+56)$ in 2015 .

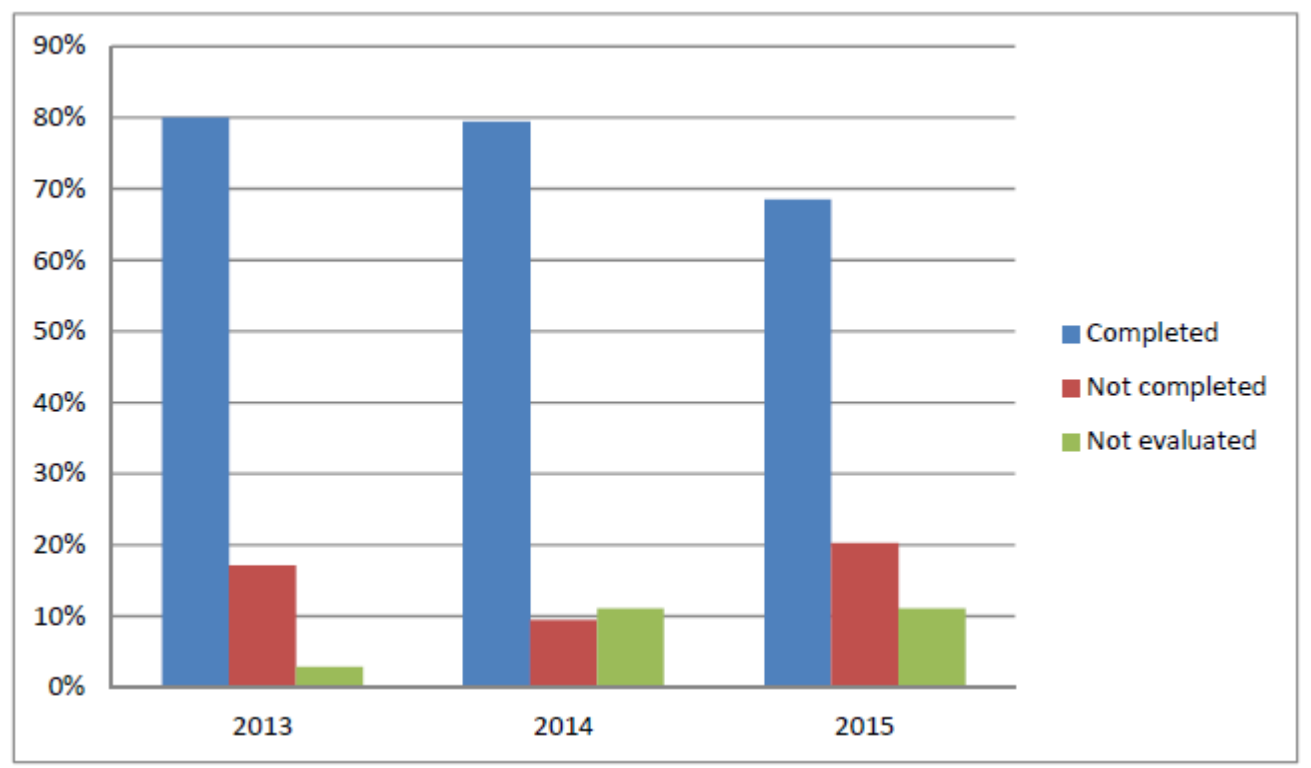

Figure 1. Comparative trends in treatment completeness, 2013-2015

The figure 1 reveals that between $75 \%$ and $80 \%$ of TB patients have completed treatment for the period of 2013 and 2014. However, in 2015, this proportion declines below 70\%, and therefore explaining the low success rate for this period.

The proportion of patients who did not complete treatment is less than $10 \%$ in 2014, whereas in 2013 and 2015 , it is between $10 \%$ and $20 \%$. 
DOI: $10.21522 / \mathrm{TIJPH} .2013 .05 .04 . \mathrm{Art} 036$

ISSN: $2520-3134$

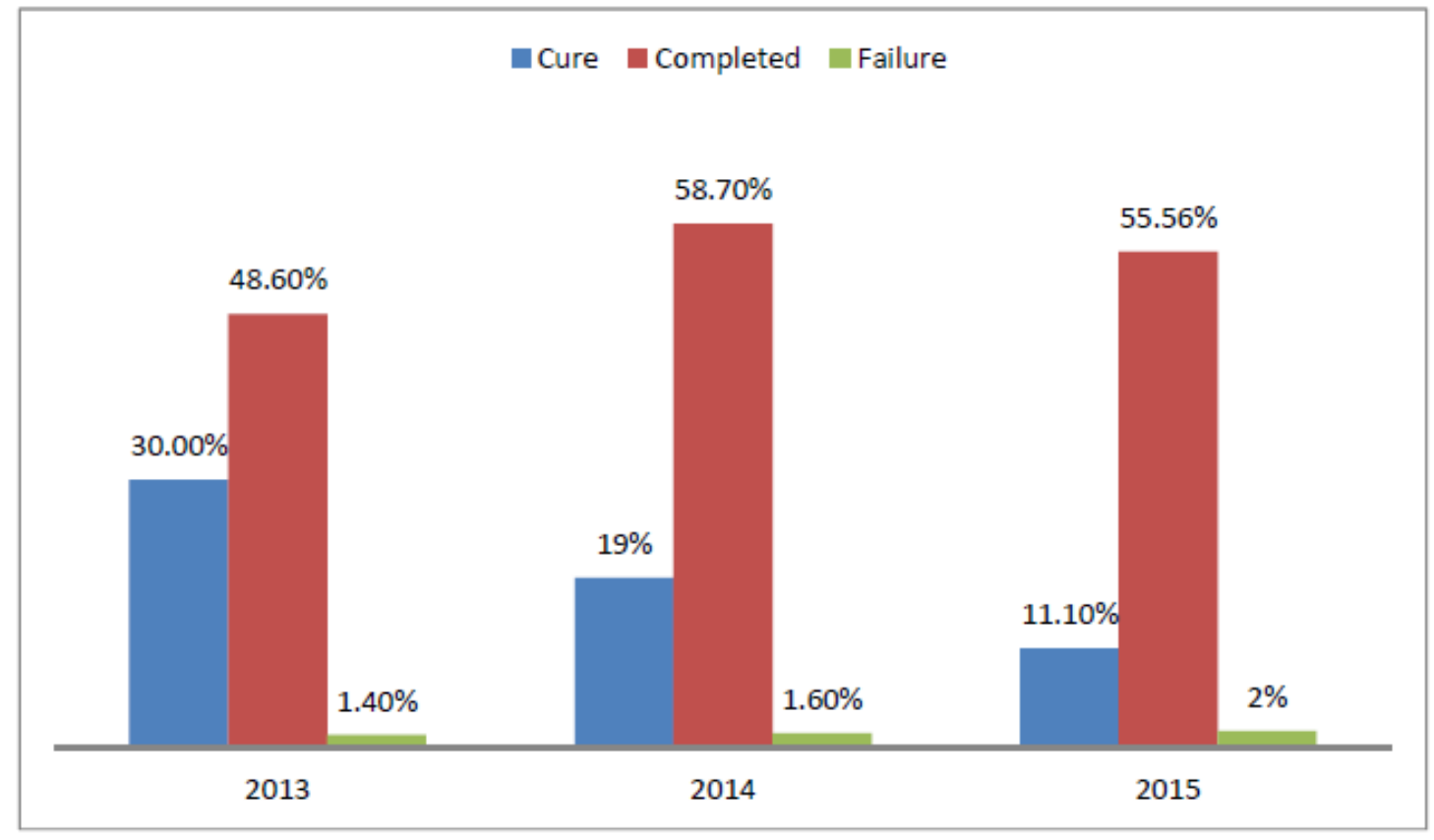

Figure 2. Comparative trends by completed treatment, 2013-2015

It appears that the cure rate ranges between 10 and $30 \%$, whereas the treatment failure remains below $3 \%$ for the whole period under evaluation.

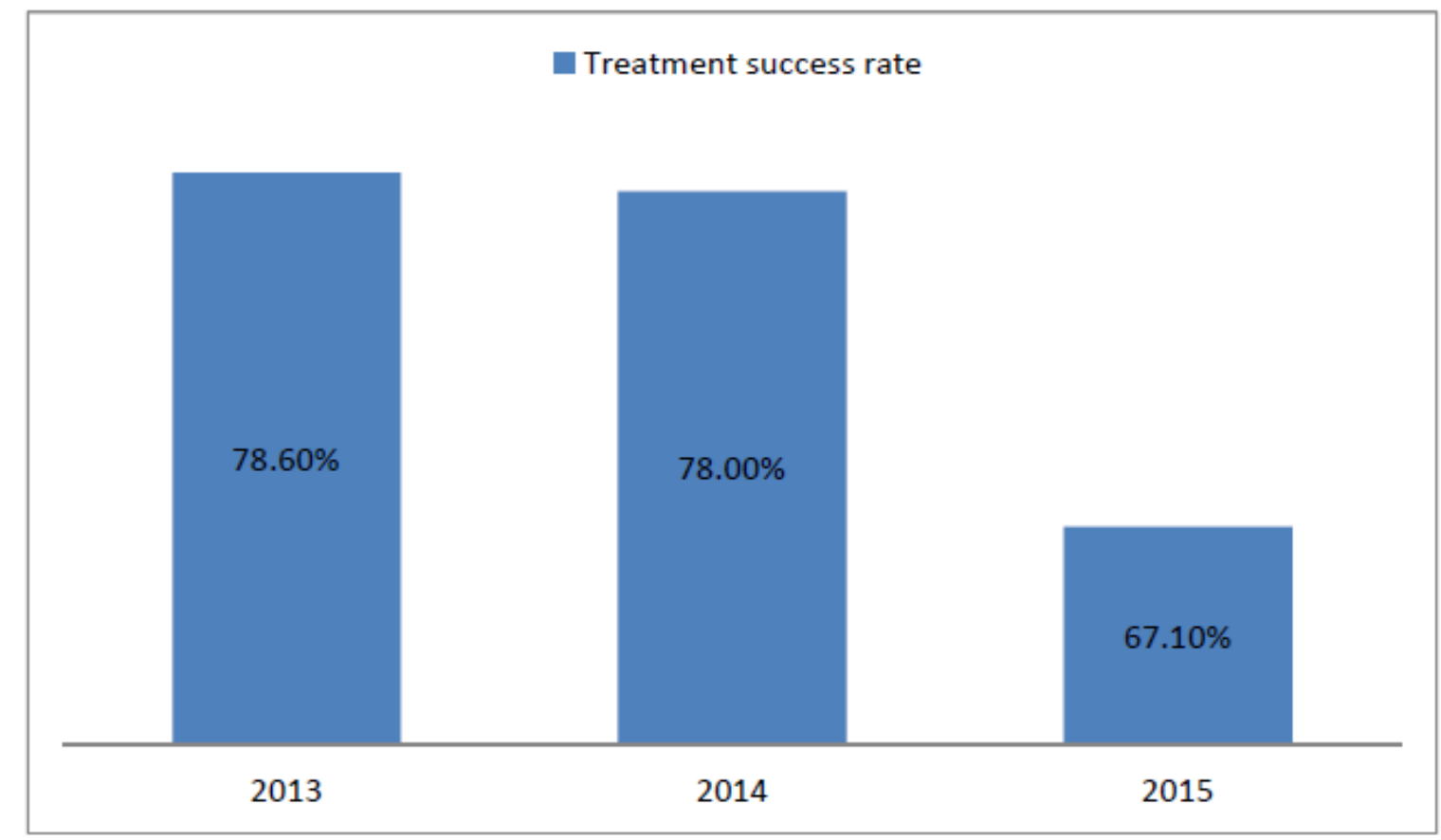

Figure 3. Comparative trends in TB treatment success rate, 2013-2015

This figure presents the comparative success rate situation for the period of 2013 to 2015. The success rate is markedly high over the first the two years of the study, but there is a decline in 2015 probably because of no documented treatment outcomes, and low cure rate. 


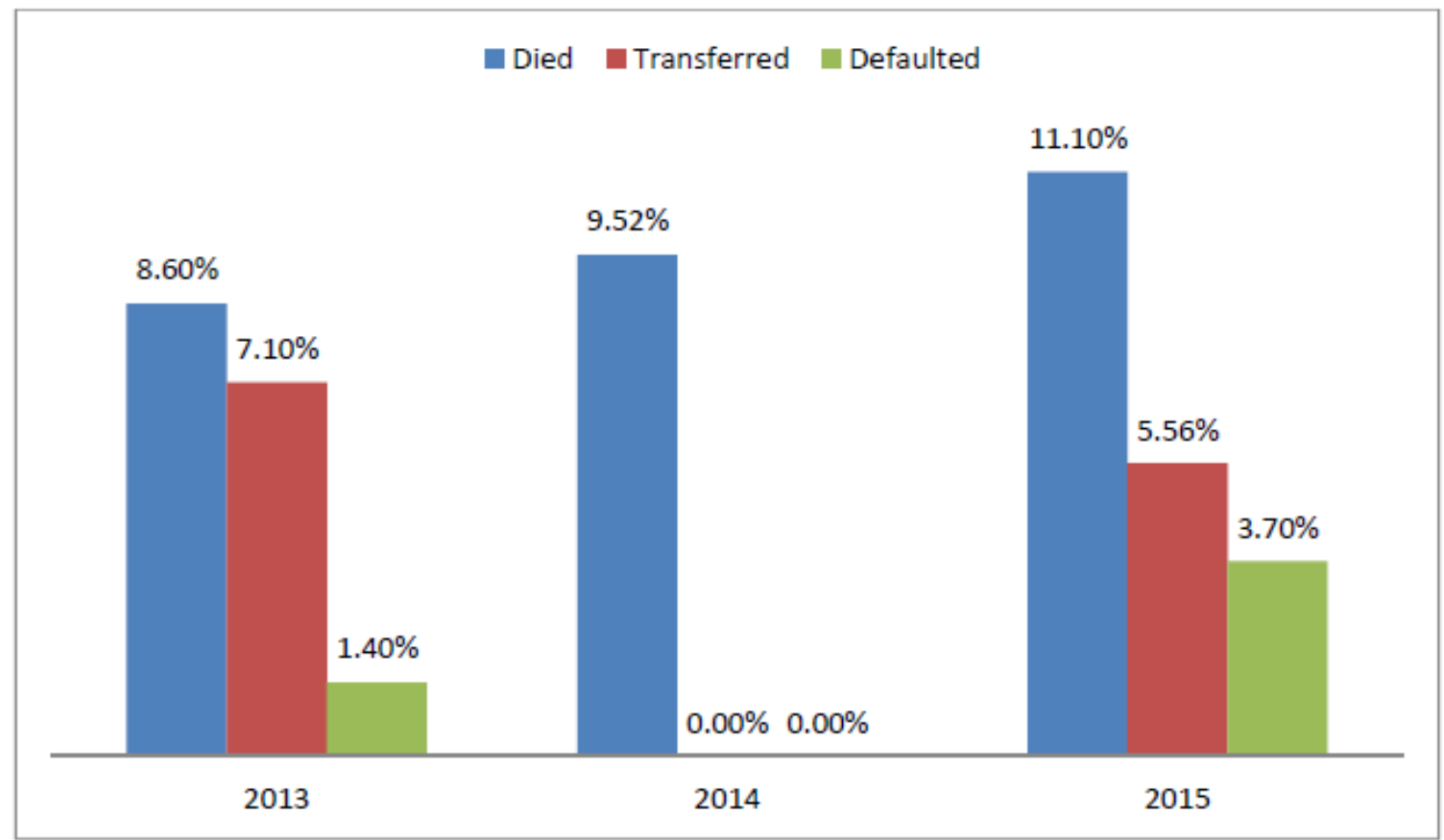

Figure 4. Comparative trends in treatment not completed, 2013-2015

The default rate remains below $5 \%$ during the evaluation period.

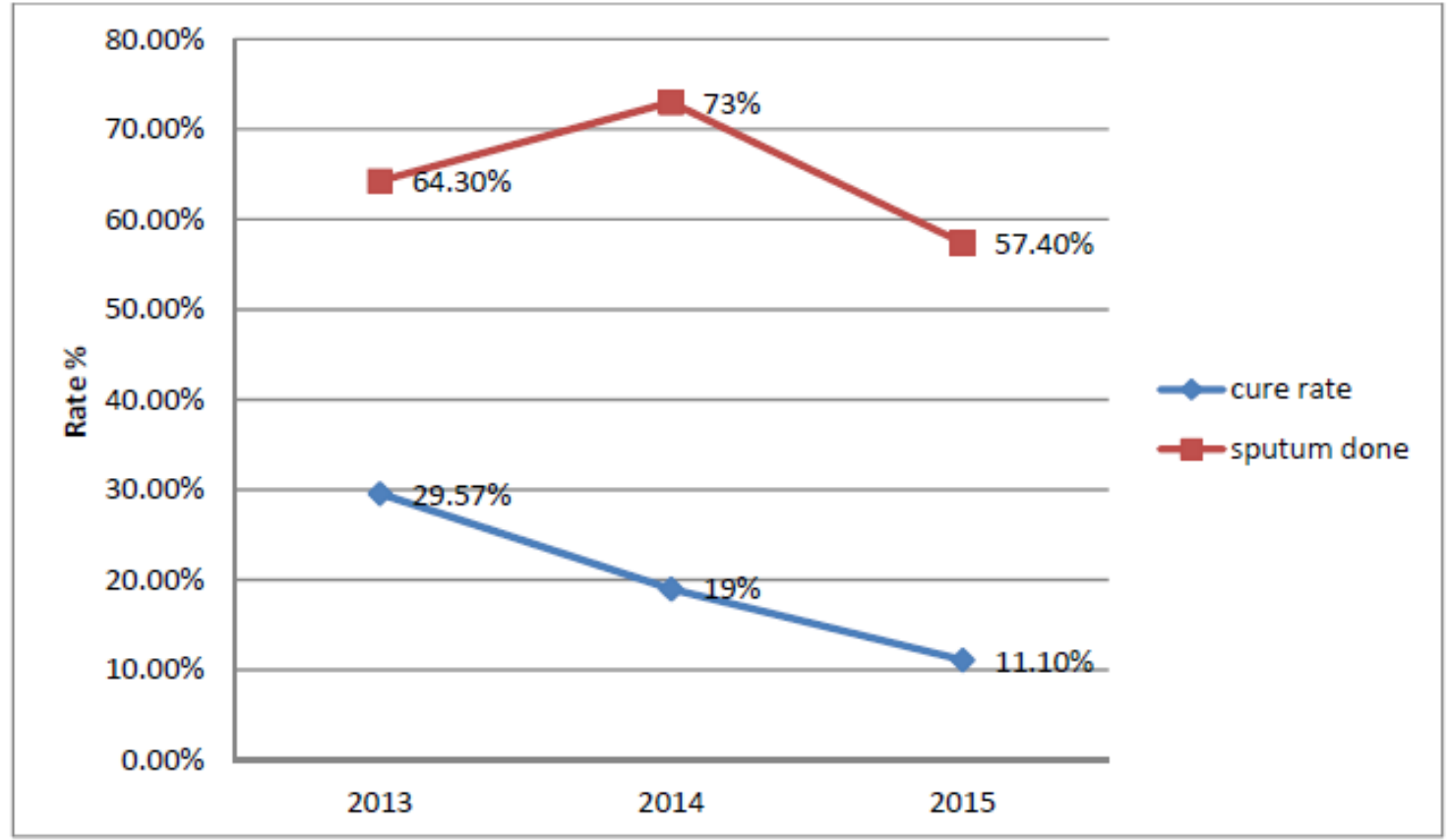

Figure 5. Comparative trends in cure rate and sputum collection, 2013-2015

This figure indicates that the cure rate does not follow the sputum collection. This situation might be due to poor sputum collection during treatment, extra pulmonary TB or poor documentation. 
DOI: $10.21522 / \mathrm{TIJPH} .2013 .05 .04 . \mathrm{Art} 036$

ISSN: $2520-3134$

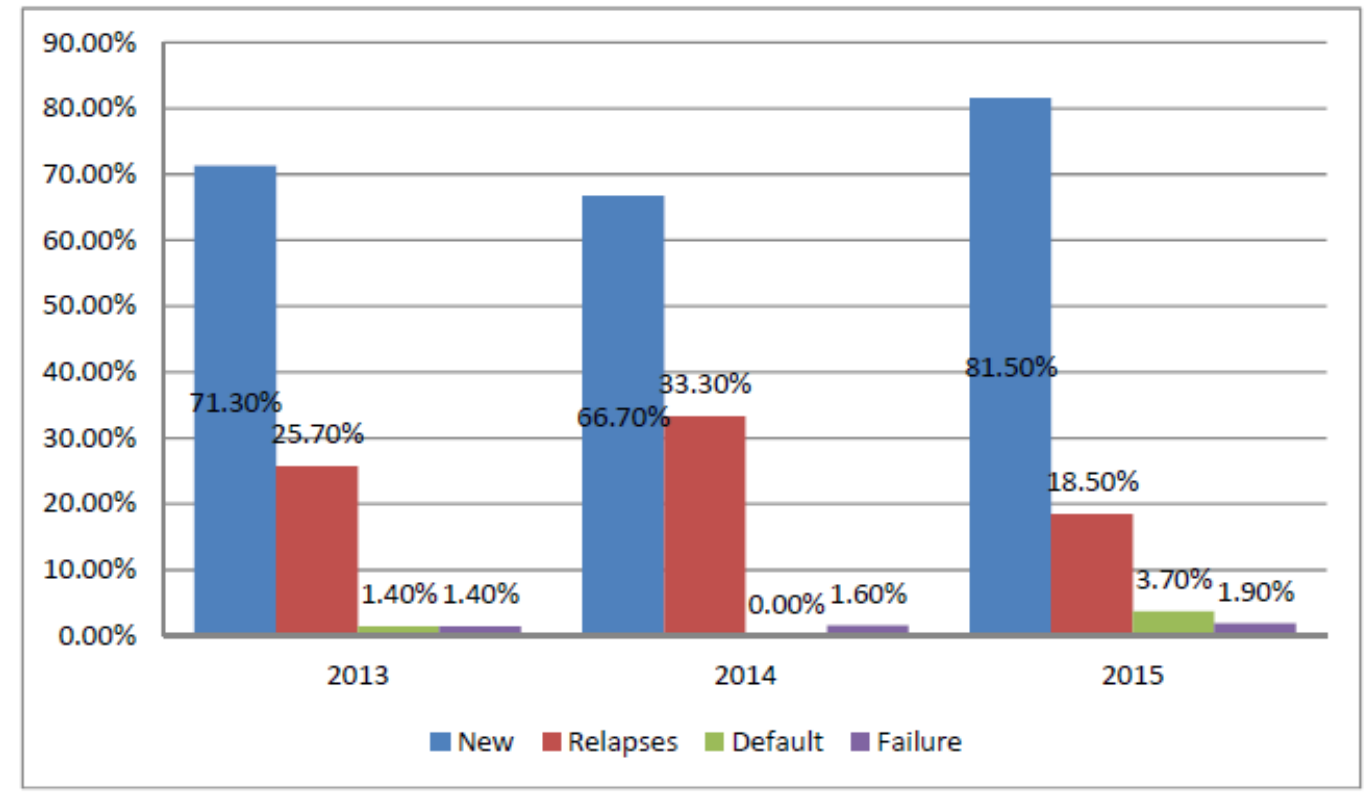

Figure 6. Comparative treatment category, 2013-2015

This figure shows that the proportion of patients who defaulted for the whole study period is below $5 \%$.

Table 4. Treatment Outcomes of TB patients by HIV status from 2013-2015

\begin{tabular}{|l|l|l|l|l|l|l|}
\hline \multirow{2}{*}{ Periods } & \multicolumn{2}{|l|}{ Known HIV status } & \multicolumn{2}{l|}{$\begin{array}{l}\text { Unknown } \\
\text { HIV status }\end{array}$} & \multicolumn{2}{l|}{ HIV positive } \\
\cline { 2 - 7 } & Tested & $\%$ & N & $\%$ & N & $\%$ \\
\hline 2013 & 70 & 100 & - & 0.0 & 40 & 57.1 \\
\hline 2014 & 57 & 90.5 & 6 & 9.52 & 31 & 50 \\
\hline 2015 & 50 & 92.6 & 4 & 7.4 & 25 & 46.3 \\
\hline
\end{tabular}

This table indicates that more $90 \%$ of TB patients know their HIV status over the whole study period. It appears that the TB/HIV co infection rate is between $40 \%$ and $60 \%$. This is well detailed in the followed figure. 


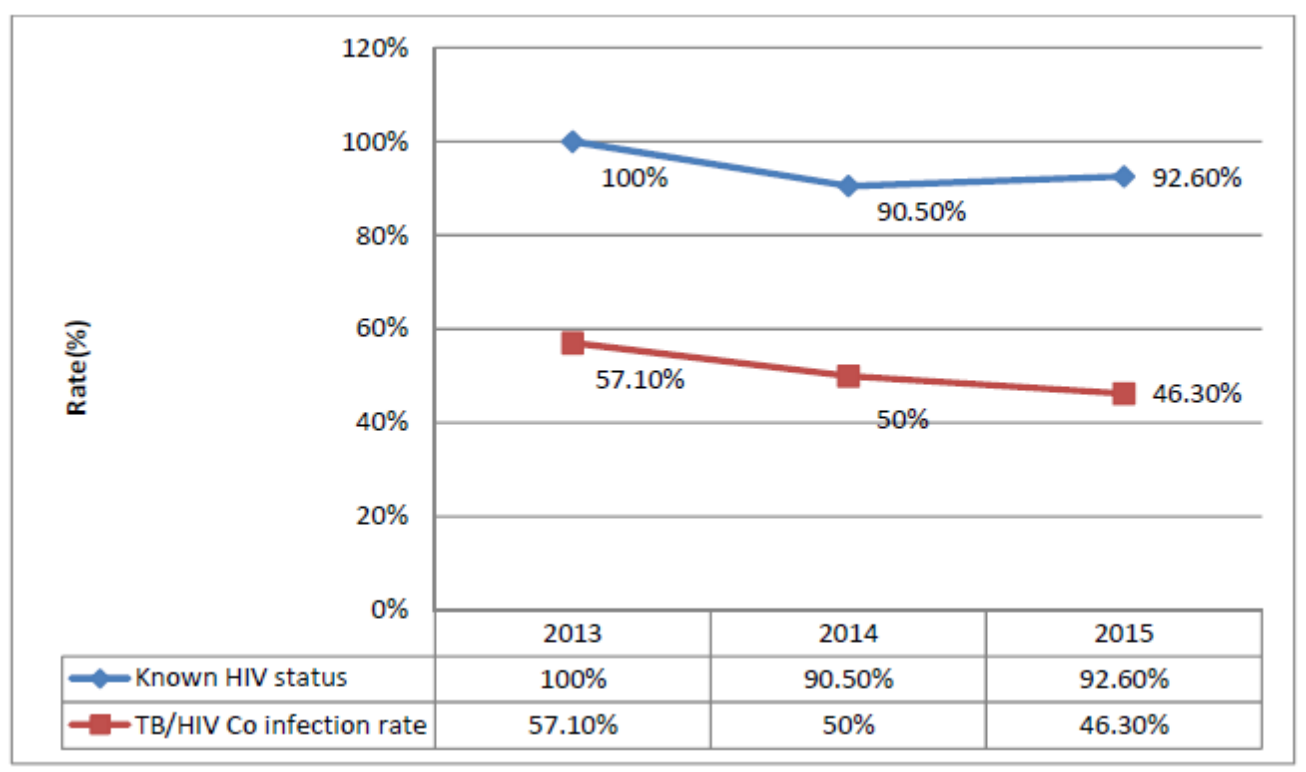

Figure 7. Trends for HIV testing for TB patients \& co infection rates, 2013-2015

This table reveals that the HIV testing rate is more than $90 \%$ over the whole study period, and the TB/HIV co infection rate is between $40-60 \%$.

The TB/HIV co infection among those who know their HIV status was between 40-60\%, proportion below the national target fixed at $60 \%$ to $80 \%$.

\section{Discussions}

\section{What is the TB cure rate?}

These results are consistent with the previous studies. In fact, this unfavorable outcome in our study concerning the cure might be due to missing sputum reexaminations during treatment or poor evaluation or documentation. It indicates a weak program.

\section{What is the proportion of patients who completed TB treatment?}

The completeness of treatment has been shown higher or very low in other parts of the Botswana. it has been reported low in most high TB burden countries.

The difference between this study and other studies could be explained by differences in sample size.

\section{What is the default rate?}

This indicated that the default rate for this period of 2013-2015 was less than 5\%. This is consistent with other published reports, such as the world's 22 high-burden countries, where this proportion ranges from less than $1 \%$ to $13 \%$.

\section{What is the TB success rate in Mabutsane sub district?}

The proportion of success rate is remarkably higher in previous studies or some parts of Botswana. Globally, the success rate was estimated at $83 \%$ in 2014, and it was $90 \%$ or more in eight of $30 \mathrm{~TB}$ burden countries, western pacific region and the Eastern Mediterranean region in 2014.

In the Southern Africa it was highest in Zambia (85\%), followed by Zimbabwe (81\%), south Africa (78\%), Lesotho (70\%), Angola (34\%) (1).

In other parts of Africa for example, Tanzania has recorded a success rate of $81 \%$ against $70 \%$ in Uganda (WHO, 2010), whereas in Ethiopia the successful outcome was 83\% for the period of 20112015(Gebretsadik Berhe et al., 2012) 
DOI: $10.21522 / \mathrm{TIJPH} .2013 .05 .04 . A r t 036$

ISSN: $2520-3134$

Overall, our findings of successful outcomes remain comparable to other researches apart from the decline mentioned in 2015 that could result from poor program performance or poor documentation.

\section{What is the proportion of TB patients with HIV test done?}

When compared to other parts of the world, the HIV testing rates are highest, but consistent with studies conducted in the same region as it has been suggested in the Southern Africa where TB is mostly HIV driven, $81 \%$ of TB patients had a notified HIV test result (WHO, 2015).

This is a weak program as shown by a low cure rates, and sputum follow ups.

\section{Acknowledgements}

This information was the result of the contribution or efforts of others in making this work project a TB performance evaluation guideline or a reference document for other projects.

I wish to acknowledge the Ministry of Health for allowing the project to be conducted. I am grateful to the Mabutsane sub district health, especially to the TB coordinator for availing the data of interest.

Finally, I would to thank Dr Bonaparte Nkomo (PHS) for his remarkable guidance during the research.

\section{References}

[1]. Botswana National TB register, 2008 89. TB and Malaria in SADC Countries. Thuthula Balfour SADC Health Sector Coordinating Unit, Pretoria.

[2]. Botswana Tuberculosis and Leprosy Programme, Annual Report 201223.

[3]. Botswana National Tuberculosis Programme Manual, Seventh Edition 2011.

[4]. Caholo J. S. Regional Integration in the Context of HIV and AIDS, TB and Malaria: The case of SADC, Presentation at the XIX International AIDS Conference, Washington DC, 2012.

[5]. Global tuberculosis report, 2015.

[6]. Global tuberculosis report, 2016 http://www.tbfacts.org/tbstatistics/\#sthash.Rn1Of6xY.dpuf

[7]. Gebretsadik Berhe et al. (2012). Treatment outcome of smear-positive pulmonary tuberculosis patients in Tigray Region, Northern Ethiopia. Group Division of Tuberculosis Elimination National Center for HIV, STD, and TB, 2006.

[8]. https://en.wikipedia.org/wiki/Southern_District_\%28Botswana\%29

[9]. Harmonized Minimum Standards for the Prevention, Treatment and Management of Tuberculosis in the SADC Region, 2010.

[10]. LIZ King and Shama ahuja. TB and HIV co infection: current trends and treatment updates, 2006.

[11]. Margaret E. Kruk et al. Timing of default from tuberculosis treatment: a systematic review, 2008. Monitoring and evaluation frameworks and the performance and governance of international funds, 2001.

[12]. Norgbe, G.K. et al. Factors influencing default rates of tuberculosis patients in Ghana, 2011.

[13]. Reid A. et al. (2006). Towards universal access to HIV prevention, treatment and support. http://www.aho.afro.who.int/profiles_information/index.php/Botswana:Tuberculosis Botswana Census, 2011.

[14]. Rebecca Gross, M.A. and Christina Blumel, Literature Review of Tuberculosis (TB) Intervention Studies, 2008

[15]. Tom Hiatt et al. (2013) Epidemiology and control of tuberculosis in the Western Pacific Region, WHO Regional Office for the Western Pacific, Manila, Philippines.

[16]. Treatment of Tuberculosis, Guidelines, fourth edition. WHO, 2010.

[17]. TB Program Evaluation Handbook: Introduction to Program Evaluation Tuberculosis Evaluation Work 